\title{
Optimizing Reinforcement
}

\author{
Structural Analysis, Challenges and Strategies \\ Adam Orlinski $^{1}$, Klaas De Rycke ${ }^{2,3}$, and Moritz Heimrath ${ }^{1}$ \\ ${ }^{1}$ University of Applied Arts Vienna, Austria \\ ${ }^{2}$ ENSAV - École nationale supérieure d'architecture de Versailles, France \\ ${ }^{3}$ Bartlett School of Architecture - UCL, London
}

\begin{abstract}
The combination of parametric modelling with structural analysis prompted new synergies between designing and engineering. The connection of structural calculation models such as Karamba3d [1] to algorithmic modelling platforms such as Grasshopper3d allowed to embed tools for analysis and simulation into the environment of creative design processes.
\end{abstract}

This way structural analysis advanced from its single sided calculation "duty" towards participation in design language of articulated structural expression.

$3 d$-concrete-printing serves as a great new territory to apply the potential of real-time parametric structural analysis in the built environment of rapid prototyping and robotic fabrication. While 3d-printed-concrete rapidly advanced in technology and empiric know-how, so also grew the ambition to utilize them for larger purposes and bigger building projects.

Known requirements such as structural integrity according to building code, interfaces for construction or waterproofing pose clear challenges for further realizations.

In order to develop 3d-concrete-printing to its full potential all challenges must serve as opportunities to dissect and rethink established norms and practices, and to construct a new interdisciplinary rule book for an emerging building technology. In this field working through challenges via prototypes serve as great basis for development and allows for an applied discourse within the wider community.

Furthermore to address challenges a flexible toolbox for structural analysis such as Karamba3d [1] offers the potential to serve as an open instrument for structural analysis and to promote $3 d$-concrete-printing within that interdisciplinary effort to its next level. On the one hand the tool enables to suggest customized and optimized rebar layouts which could support the development of 3D-concrete-printing further. By enabling this more customizable calculation and tailored real time feedback for complex structures, solutions can develop more structurally informed. On the other hand, recent developments with Karamba3D within our office of Bollinger+Grohmann have shown the possibility of real time feedback loops between the physical printing process and the digital calculation model.

Both developments show the potential that material systems can be optimized towards specific patterns or values of forces, or structures can be evaluated in real time for their load bearing capacity while hardening during printing.

Keywords: Optimization, Parametric Engineering, Force-Flow, 3d-Concrete-Printing 

Structures"

Conference presentation video: https://doi.org/10.5446/1766

\section{Competing interests}

The author declares no competing interests.

\section{References}

1. Preisinger, C. (2013), Linking Structure and Parametric Geometry. Architectural Design, 83: 110-113 DOI: https://doi.org/10.1002/ad.1564. 